Georgian Mathematical Journal

Volume 14 (2007), Number 2, 325-340

\title{
SINGULAR DIRICHLET BOUNDARY VALUE PROBLEM FOR SECOND ORDER ODE
}

\author{
IRENA RACHŮNKOVÁ AND JAKUB STRYJA
}

Cordially dedicated to Professor Ivan Kiguradze

on his 70th birthday anniversary

\begin{abstract}
This paper investigates the singular Dirichlet problem

$$
-u^{\prime \prime}=f\left(t, u, u^{\prime}\right), \quad u(0)=0, u(T)=0,
$$

where $f$ satisfies the Carathéodory conditions on the set $(0, T) \times \mathbb{R}_{0}^{2}$ and $\mathbb{R}_{0}=\mathbb{R} \backslash\{0\}$.

The function $f(t, x, y)$ can have time singularities at $t=0$ and $t=T$ and space singularities at $x=0$ and $y=0$. The existence principle for the above problem is given and its application is presented here. The paper provides conditions which guarantee the existence of a solution which is positive on $(0, T)$ and which has the absolutely continuous first derivative on $[0, T]$.
\end{abstract}

2000 Mathematics Subject Classification: 34B16, 34B15, 34B18.

Key words and phrases: Singular Dirichlet problem, existence, smooth positive solution, time and space singularities.

\section{INTRODUCTION}

Let $[0, T] \subset \mathbb{R}$ and $\mathbb{R}_{0}=\mathbb{R} \backslash\{0\}$. We will investigate the solvability of the problem

$$
\begin{gathered}
-u^{\prime \prime}=f\left(t, u, u^{\prime}\right), \\
u(0)=0, \quad u(T)=0,
\end{gathered}
$$

where $f$ satisfies the Carathéodory conditions on $(0, T) \times \mathbb{R}_{0}^{2}$ and $f(t, x, y)$ can have time singularities at $t=0, t=T$ and space singularities at $x=0$ and $y=0$.

Definition 1.1. We say that $f$ has a time singularity at $t=0(t=T)$ if there exist $x, y \in \mathbb{R}_{0}$ such that

$$
\int_{0}^{\varepsilon}|f(t, x, y)| \mathrm{d} t=\infty \quad\left(\int_{T-\varepsilon}^{T}|f(t, x, y)| \mathrm{d} t=\infty\right)
$$

for any sufficiently small $\varepsilon>0$.

Definition 1.2. We say that $f$ has a space singularity at $x=0(y=0)$ if there exists a set $J \subset[0, T]$ with a positive Lebesgue measure such that the 
condition

$$
\limsup _{x \rightarrow 0}|f(t, x, y)|=\infty \quad\left(\limsup _{y \rightarrow 0}|f(t, x, y)|=\infty\right)
$$

holds for a.e. $t \in J$ and some $y \in \mathbb{R}_{0}\left(x \in \mathbb{R}_{0}\right)$.

In what follows we will use the notation:

$[a, b] \subset \mathbb{R} ; \Sigma \subset(a, b)$ is a finite set; $\mathcal{M} \subset \mathbb{R}^{2}$;

$C[a, b]$ is the Banach space of functions continuous on $[a, b]$ with the norm $\|x\|_{C}=\max \{|x(t)| ; t \in[a, b]\}$;

$C^{1}[a, b]$ is the Banach space of functions having continuous first derivatives on $[a, b]$ with the norm $\|x\|_{C^{1}}=\|x\|_{C}+\left\|x^{\prime}\right\|_{C}$;

$A C^{1}[a, b]$ is the set of functions having absolutely continuous derivatives on $[a, b]$

$A C_{l o c}^{1}((a, b) \backslash \Sigma)$ is the set of functions $x \in A C^{1}[c, d]$ for each $[c, d] \subset[a, b] \backslash \Sigma$; $L[a, b]$ is the Banach space of functions Lebesgue integrable on $[a, b]$ with the norm $\|x\|_{L}=\int_{a}^{b}|x(t)| \mathrm{d} t$;

$\operatorname{Car}([a, b] \times \mathcal{M})$ is the set of functions $f:[a, b] \times \mathcal{M} \rightarrow \mathbb{R}$ satisfying the Carathéodory conditions on $[a, b] \times \mathcal{M}$, i.e.,

$$
\begin{aligned}
& f(\cdot, x, y):[a, b] \rightarrow \mathbb{R} \text { is measurable for all }(x, y) \in \mathcal{M} ; \\
& f(t, \cdot, \cdot): \mathcal{M} \rightarrow \mathbb{R} \text { is continuous for a.e. } t \in[a, b]
\end{aligned}
$$

for each compact set $\mathcal{K} \subset \mathcal{M}$ there is a function $m_{\mathcal{K}} \in L[a, b]$ such that

$$
|f(t, x, y)| \leq m_{\mathcal{K}}(t) \text { for a.e. } t \in[a, b] \text { and all }(x, y) \in \mathcal{K} .
$$

$\operatorname{Car}((a, b) \times \mathcal{M})$ is the set of functions $f \in \operatorname{Car}([c, d] \times \mathcal{M})$ for each $[c, d] \subset$ $(a, b)$;

meas $\mathcal{A}$ is the Lebegue measure of $\mathcal{A} \subset \mathbb{R}$.

We say that a sequence $\left\{v_{n}\right\} \subset C[0, T]$ is equicontinuous on $[0, T]$ if for each $\varepsilon>0$ there exists $\delta>0$ such that for each $t_{1}, t_{2} \in[0, T]$

$$
\left|t_{1}-t_{2}\right|<\delta \Rightarrow\left|v_{n}\left(t_{1}\right)-v_{n}\left(t_{2}\right)\right|<\varepsilon
$$

for each $n \in \mathbb{N}$.

Definition 1.3. By a solution of problem (1.1), (1.2) we understand a function $u \in A C^{1}[0, T]$ satisfying equation (1.1) a.e. on $[0, T]$ and satisfying conditions (1.2).

In literature we can find an alternative approach to the solvability of singular problems where solutions are defined as continuous functions whose first derivatives can have discontinuities at some points in $[0, T]$. Here we will call such functions $w$-solutions and according to [4] or [11] we will define them as follows:

Definition 1.4. We say that $u \in C[0, T]$ is a w-solution of $(1.1),(1.2)$ if there exists a finite set $\Sigma \subset(0, T)$ such that $u \in A C_{l o c}^{1}((0, T) \backslash \Sigma)$ satisfies equation (1.1) a.e. on $[0, T]$ and satisfies conditions (1.2). 
A systematic study of the solvability of Dirichlet problems having both time and space singularities was initiated by Taliaferro [21]. Now, we can find a large group of works which focused their attention on the existence of $w$-solutions, e.g. [1]-[4], [10]-[15], and a less number of works which provide also conditions for the existence of solutions, e.g. [5], [7], [8], [16], [17], [22]. All the above works deal with differential equations where the nonlinearity $f(t, x, y)$ has a space singularity at $x=0$ and/or time singularities at $t=0, t=T$. The first existence result for the Dirichlet problem where $f(t, x, y)$ has singularities at both variables $x$ and $y$ was obtained by Staněk [20]. He assumed that $f$ is strictly positive and its behaviour in a right neighbourhood of the singular point $x=0$ is controlled by a function $\omega_{0}(x)$ which is integrable. Then we say that $f$ has a weak space singularity at $x=0$. Here, we extend the existence result of [20] to $f$ with a strong space singularity at $x=0$, i.e., we consider $f$ which is controlled by a nonintegrable function $\omega_{0}(x)$. Our main result is Theorem 2.2.

In our proofs we will need the following Fredholm type existence theorem:

Theorem 1.5 (Fredholm type existence theorem, [23]). Let $h \in \operatorname{Car}([a, b] \times$ $\mathbb{R}^{2}$ ) and $m \in L[a, b]$ be such that

$$
|h(t, x, y)| \leq m(t) \text { for a.e. } t \in[a, b] \text { and all } x, y \in \mathbb{R} \text {. }
$$

Then the problem

$$
-u^{\prime \prime}=h\left(t, u, u^{\prime}\right), \quad u(a)=u(b)=0
$$

has a solution $u \in A C^{1}[a, b]$.

We approximate the singular equation (1.1) by a sequence of regular equations

$$
-u^{\prime \prime}=f_{n}\left(t, u, u^{\prime}\right) \text {, }
$$

where $f_{n} \in \operatorname{Car}\left([0, T] \times \mathbb{R}^{2}\right), n \in \mathbb{N}$.

Having a sequence $\left\{u_{n}\right\}$ of solutions of problems (1.3), (1.2) we need to prove the existence of its converging subsequence. A type of this convergence defines the properties of its limit $u$ and, at the same time, it determines if $u$ is a solution (or a $w$-solution) of the original problem (1.1), (1.2). The investigation of convergence is based on next two theorems.

Theorem 1.6 (Arzelà-Ascoli theorem in $C[a, b]$ and $\left.C^{1}[a, b],[9]\right) . A \subset C[a, b]$ is relatively compact if and only if $A$ is bounded in $C[a, b]$ and functions in $A$ are equicontinuous on $[a, b]$.

$B \subset C^{1}[a, b]$ is relatively compact if and only if $B$ is bounded in $C^{1}[a, b]$ and the first derivatives of functions in $B$ are equicontinuous on $[a, b]$.

Theorem 1.7 (Fatou lemma, [19]). Let $\varphi_{n} \in L[a, b]$ for $n \in \mathbb{N}$ and $\lim _{n \rightarrow \infty} \varphi_{n}(t)=\varphi(t)$ a.e. on $[a, b]$. Assume that there exists $c \in(0, \infty)$ such that

$$
\int_{a}^{b}\left|\varphi_{n}(t)\right| \mathrm{d} t \leq c \text { for each } n \in \mathbb{N} \text {. }
$$


Then $|\varphi| \in L[a, b]$ and $\int_{a}^{b}|\varphi(t)| \mathrm{d} t \leq c$. (Clearly, $\varphi \in L[a, b]$, as well.)

The paper is organized as follows. In Section 2 we provide the existence principle (Theorem 2.2) giving the properties of approximating regular functions $f_{n}$ in order to find a sequence of approximate solutions converging to a solution (or to a $w$-solution) of problem (1.1), (1.2). In Section 3 we apply the existence principle of Section 2 to get conditions which are imposed on $f$ directly and yield the solvability of problem (1.1), (1.2).

\section{Existence Principle for Singular Dirichlet Problems}

Theorem 2.1. Assume that $f \in \operatorname{Car}\left((0, T) \times \mathbb{R}_{0}^{2}\right), f_{n} \in \operatorname{Car}\left([0, T] \times \mathbb{R}^{2}\right)$,

$$
\begin{gathered}
f_{n}(t, x, y)=f(t, x, y) \text { for a.e. } t \in \Delta_{n} \text { and } \\
\text { each }|x| \geq \frac{1}{n},|y| \geq \frac{1}{n}, n \in \mathbb{N}, \\
\text { where } \Delta_{n}=\left[\frac{1}{n}, T-\frac{1}{n}\right] \cap[0, T] ;
\end{gathered}
$$

there exists a bounded set $\Omega \subset C^{1}[0, T]$ such that for each

$$
n \in \mathbb{N} \text {, problem (1.3), (1.2) has a solution } u_{n} \in \Omega \text {. }
$$

Then there exist $u \in C[0, T]$ and a subsequence $\left\{u_{k}\right\} \subset\left\{u_{n}\right\}$ such that

$$
\lim _{k \rightarrow \infty}\left\|u_{k}-u\right\|_{C}=0 .
$$

Assume in addition that there exists a finite set $\Sigma=\left\{s_{1}, \ldots, s_{\nu}\right\} \subset(0, T)$ such that

on each interval $[a, b] \subset(0, T) \backslash \Sigma$ the sequence $\left\{u_{k}^{\prime}\right\}$ is equicontinuous.

Then $u \in C^{1}((0, T) \backslash \Sigma)$ and

$$
\lim _{k \rightarrow \infty} u_{k}^{\prime}(t)=u^{\prime}(t) \text { locally uniformly on }(0, T) \backslash \Sigma \text {. }
$$

Proof. By (2.2) there exist $r>0$ and a sequence $\left\{u_{n}\right\}$ of solutions of (1.3), (1.2) such that

$$
\left\|u_{n}\right\|_{C^{1}} \leq r \text { for each } n \in \mathbb{N} .
$$

Therefore the sequence $\left\{u_{n}\right\}$ is bounded in $C[0, T]$ and equicontinuous on $[0, T]$. By Theorem 1.6 (Arzelà-Ascoli) we can choose a subsequence $\left\{u_{l}\right\}$ such that

$$
\lim _{l \rightarrow \infty}\left\|u_{l}-u\right\|_{C}=0, \quad u \in C[0, T] .
$$

Now assume also (2.4) and choose an interval $[a, b] \subset(0, T) \backslash \Sigma$ arbitrarily. Then $\left\{u_{l}^{\prime}\right\}$ is equicontinuous on $[a, b]$. By (2.6) the sequence $\left\{u_{l}\right\}$ is bounded in $C^{1}[a, b]$. Theorem 1.6 implies that we can choose a subsequence $\left\{u_{k}\right\} \subset\left\{u_{l}\right\}$ such that

$$
\lim _{k \rightarrow \infty} u_{k}^{\prime}(t)=u^{\prime}(t) \text { uniformly on }[a, b] .
$$

By virtue of (2.7) the sequence $\left\{u_{k}\right\}$ satisfies (2.3). Using the diagonalization method we can choose $\left\{u_{k}\right\}$ such that (2.5) holds too. 
Theorem 2.2 (Existence principle for problem (1.1), (1.2)). Let all assumptions of Theorem 2.1 be fulfilled. Let the finite set $\Sigma$ have the form:

$$
\Sigma=\left\{s \in(0, T): u(s)=0 \text { or } u^{\prime}(s)=0 \text { or } u^{\prime}(s) \text { does not exist. }\right\}
$$

Then $u \in A C_{\text {loc }}^{1}((0, T) \backslash \Sigma)$ is a w-solution of (1.1), (1.2).

Denote $s_{0}=0$ and $s_{\nu+1}=T$. If $u$ and $\left\{u_{k}\right\}$ satisfy (2.3), (2.5) and, besides, there exist $\eta \in\left(0, \frac{T}{2}\right), \lambda_{0}, \mu_{0}, \lambda_{1}, \mu_{1}, \ldots, \lambda_{\nu+1}, \mu_{\nu+1} \in\{-1,1\}$ and $\psi \in L[0, T]$ such that

$$
\begin{gathered}
\lambda_{i} f_{k}\left(t, u_{k}(t), u_{k}^{\prime}(t)\right) \geq \psi(t) \text { for a.e. } t \in\left(s_{i}-\eta, s_{i}\right) \cap(0, T) \\
\mu_{i} f_{k}\left(t, u_{k}(t), u_{k}^{\prime}(t)\right) \geq \psi(t) \text { for a.e. } t \in\left(s_{i}, s_{i}+\eta\right) \cap(0, T), \\
\text { for all } i \in\{0, \ldots \nu+1\}, \quad k \in \mathbb{N},
\end{gathered}
$$

then $u \in A C^{1}[0, T]$ is a solution of (1.1), (1.2).

Proof. 1. Let (2.1), (2.2), (2.4) and (2.8) be true. Then for $k \in \mathbb{N}$

$$
\begin{gathered}
-u_{k}^{\prime \prime}(t)=f_{k}\left(t, u_{k}(t), u_{k}^{\prime}(t)\right) \text { for a.e. } t \in[0, T], \\
u_{k}(0)=0, \quad u_{k}(T)=0,
\end{gathered}
$$

and by Theorem 2.1, there exists $u \in C[0, T]$ such that (2.3) and (2.5) hold. By (2.3), $u$ satisfies (1.2).

Define the sets

$$
\begin{gathered}
V_{1}=\left\{t \in(0, T): f(t, \cdot, \cdot): \mathbb{R}_{0}^{2} \rightarrow \mathbb{R} \text { is not continuous }\right\}, \\
V_{2}=\{t \in(0, T): \text { the equality in }(2.1) \text { is not satisfied }\}
\end{gathered}
$$

and let

$$
U=(0, T) \backslash\left(\Sigma \cup V_{1} \cup V_{2}\right)
$$

We see that

$$
\operatorname{meas}\left(\Sigma \cup V_{1} \cup V_{2}\right)=0 \text {. }
$$

Choose an arbitrary $t \in U$. Then there exists $k_{0} \in \mathbb{N}$, such that for each $k \in \mathbb{N}$, $k \geq k_{0}$ :

and

$$
t \in \Delta_{k}, \quad\left|u_{k}(t)\right|>\frac{1}{k}, \quad\left|u_{k}^{\prime}(t)\right|>\frac{1}{k}
$$

$$
f_{k}\left(t, u_{k}(t), u_{k}^{\prime}(t)\right)=f\left(t, u_{k}(t), u_{k}^{\prime}(t)\right) .
$$

Since $t$ is an arbitrary element of $U$, by (2.3), (2.5) and (2.11) we get

$$
\lim _{k \rightarrow \infty} f_{k}\left(t, u_{k}(t), u_{k}^{\prime}(t)\right)=f\left(t, u(t), u^{\prime}(t)\right) \text { a.e. on }[0, T] \text {. }
$$

Now choose an arbitrary interval $[a, b] \subset(0, T) \backslash \Sigma$ and integrate equation (2.10). We get

$$
-u_{k}^{\prime}(t)+u_{k}^{\prime}(a)=\int_{a}^{t} f_{k}\left(s, u_{k}(s), u_{k}^{\prime}(s)\right) \mathrm{d} s \text { for each } t \in[a, b] .
$$

Moreover, there exists $k^{*} \in \mathbb{N}$ such that for each $k \in \mathbb{N}, k \geq k^{*}$

$$
\left|f_{k}\left(t, u_{k}(t), u_{k}^{\prime}(t)\right)\right| \leq m(t) \text { for a.e. } t \in[a, b],
$$


where

$$
m(t)=\sup \left\{|f(t, x, y)|: \frac{1}{k^{*}} \leq|x| \leq r, \quad \frac{1}{k^{*}} \leq|y| \leq r\right\} \in L[a, b] .
$$

Since $m \in L[a, b]$ we can apply Lebesgue convergence theorem on $[a, b]$ and get $f\left(\cdot, u(\cdot), u^{\prime}(\cdot)\right) \in L[a, b]$. Moreover,

$$
\lim _{k \rightarrow \infty} \int_{a}^{b} f_{k}\left(s, u_{k}(s), u_{k}^{\prime}(s)\right) \mathrm{d} s=\int_{a}^{b} f\left(s, u(s), u^{\prime}(s)\right) \mathrm{d} s,
$$

which by (2.13) yields.

$$
-u^{\prime}(t)+u^{\prime}(a)=\int_{a}^{t} f\left(s, u(s), u^{\prime}(s)\right) \mathrm{d} s \text { for each } t \in[a, b] .
$$

Since $[a, b]$ is an arbitrary interval in $(0, T) \backslash \Sigma$, we get that $u \in A C_{l o c}^{1}((0, T) \backslash \Sigma)$ is a $w$-solution of (1.1), (1.2).

2. Now assume that there exist $\eta \in\left(0, \frac{T}{2}\right), \lambda_{0}, \mu_{0}, \lambda_{1}, \mu_{1}, \ldots, \lambda_{\nu+1}, \mu_{\nu+1} \in$ $\{-1,1\}$ and $\psi \in L[0, T]$ such that (2.9) holds. Since $u$ is a $w$-solution of (1.1), (1.2), it remains to prove that $u \in A C^{1}[0, T]$.

Choose $i \in\{0, \ldots, \nu+1\}$ and denote $\left(c_{i}, d_{i}\right)=\left(s_{i}-\eta, s_{i}\right) \cap(0, T)$. For $k \in \mathbb{N}$ and for a.e. $t \in\left(c_{i}, d_{i}\right)$ we denote

$$
h_{k}(t)=\lambda_{i} f_{k}\left(t, u_{k}(t), u_{k}^{\prime}(t)\right)+|\psi(t)|, \quad h(t)=\lambda_{i} f\left(t, u(t), u^{\prime}(t)\right)+|\psi(t)| .
$$

Then $h_{k} \in L\left[c_{i}, d_{i}\right]$ and according to $(2.12)$ we have

$$
\lim _{k \rightarrow \infty} h_{k}(t)=h(t) \text { for a.e. } t \in\left[c_{i}, d_{i}\right] \text {. }
$$

Integrating $(2.10)$ on $\left[c_{i}, d_{i}\right]$ we get

$$
\int_{c_{i}}^{d_{i}} f_{k}\left(s, u_{k}(s), u_{k}^{\prime}(s)\right) \mathrm{d} s=-u_{k}^{\prime}\left(d_{i}\right)+u_{k}^{\prime}\left(c_{i}\right)
$$

Therefore, by (2.6) and (2.9)

$$
\begin{aligned}
\int_{c_{i}}^{d_{i}}\left|h_{k}(s)\right| \mathrm{d} s= & \int_{c_{i}}^{d_{i}} h_{k}(s) \mathrm{d} s=\lambda_{i} \int_{c_{i}}^{d_{i}} f_{k}\left(s, u_{k}(s), u_{k}^{\prime}(s)\right) \mathrm{d} s \\
& +\int_{c_{i}}^{d_{i}}|\psi(s)| \mathrm{d} s \leq\left|u_{k}^{\prime}\left(d_{i}\right)\right|+\left|u_{k}^{\prime}\left(c_{i}\right)\right|+\int_{c_{i}}^{d_{i}}|\psi(s)| \mathrm{d} s \leq c,
\end{aligned}
$$

where $c=2 r+\|\psi\|_{L}$.

Theorem 1.7 (Fatou) implies that $h \in L\left[c_{i}, d_{i}\right]$ and $f\left(\cdot, u(\cdot), u^{\prime}(\cdot)\right) \in L\left[c_{i}, d_{i}\right]$.

If $\left(c_{i}, d_{i}\right)=\left(s_{i}, s_{i}+\eta\right) \cap(0, T)$ we argue similarly. 
Hence $f\left(\cdot, u(\cdot), u^{\prime}(\cdot)\right) \in L[0, T]$ and the equality in $(2.14)$ is fulfilled for each $t \in[0, T]$ and $u \in A C^{1}[0, T]$. We have proved that $u$ is a solution of (1.1), $(1.2)$.

\section{Application of Existence Principle}

The main result of this section is Theorem 3.1, where we present the conditions sufficient for the existence of a solution of problem (1.1), (1.2) which is positive on $(0, T)$.

Now, let us state our assumptions on problem (1.1), (1.2). We are interested in the existence of a positive solution and hence we investigate problem (1.1), $(1.2)$ on the set $[0, T] \times[0, \infty) \times \mathbb{R}$. Denote $\mathcal{D}=(0, \infty) \times \mathbb{R}_{0}$. We assume that $f \in \operatorname{Car}([0, T] \times \mathcal{D})$ has space singularities at $x=0$ and $y=0$, particularly

$$
\begin{cases}\limsup _{x \rightarrow 0+} f(t, x, y)=\infty & \text { for a.e. } t \in[0, T] \text { and for some } y \in \mathbb{R}_{0}, \\ \limsup _{y \rightarrow 0} f(t, x, y)=\infty & \text { for a.e. } t \in[0, T] \text { and for some } x \in(0, \infty) .\end{cases}
$$

Theorem 3.1. Let (3.1) hold and let $c, \gamma, \delta \in(0, \infty), \alpha, \beta \in[0,1]$. Assume that there exist positive and nonincreasing functions $\omega_{0}, \omega_{1} \in C(0, \infty)$ and nonnegative functions $h_{0}, h_{1}, h_{2} \in L[0, T]$ satisfying

$$
\begin{aligned}
\int_{0}^{T}\left(t^{\gamma}+t^{\delta}\right) \omega_{0}(t) \mathrm{d} t<\infty, & \int_{0}^{T} \omega_{1}(t) \mathrm{d} t<\infty . \\
T\left\|h_{1}\right\|_{L}+\left\|h_{2}\right\|_{L}<1 & \text { for } \alpha, \beta=1 ; \\
T\left\|h_{1}\right\|_{L}<1 & \text { for } \alpha=1, \beta<1 ; \\
\left\|h_{2}\right\|_{L}<1 & \text { for } \alpha<1, \beta=1 ; \\
c \leq f(t, x, y) \leq t^{\gamma}(T-t)^{\delta} \omega_{0}(x)+\omega_{1}(|y|)+h_{0}(t)+h_{1}(t) x^{\alpha}+h_{2}(t)|y|^{\beta} & \\
\text { for } \text { a.e. } t \in[0, T], & \text { and all } x \in(0, \infty), \quad y \in \mathbb{R}_{0} .
\end{aligned}
$$

Then problem (1.1), (1.2) has a solution positive on $(0, T)$.

The proof of Theorem 3.1 is based on the Existence principle (Theorem 2.2), where the existence of a bounded set $\Omega$ is necessary. Therefore we first prove a priori estimates for a class of functions which will be needed for the construction of such a set $\Omega$.

Lemma 3.2. Let $c>0$. Then there exists $\eta>0$ such that for each $u \in$ $A C^{1}[0, T]$ satisfying $(1.2)$ and

$$
c \leq-u^{\prime \prime}(t) \text { for a.e. } t \in[0, T],
$$

the estimate $\|u\|_{C} \geq \eta$ is valid.

Proof. Let $G(t, s)$ be the Green function of problem $(1.2),-u^{\prime \prime}(t)=0$.

Then

$$
G(t, s)= \begin{cases}\frac{t(T-s)}{T}, & t \leq s \\ \frac{s(T-t)}{T}, & s \leq t\end{cases}
$$


We define

$$
\Phi(t, s)=\frac{G(t, s)}{t(T-t)} \quad \text { for } \quad(t, s) \in(0, T) \times(0, T) .
$$

For any $s \in(0, T)$ we have

$$
\begin{aligned}
& \lim _{t \rightarrow 0+} \Phi(t, s)=\lim _{t \rightarrow 0+} \frac{t(T-s)}{t(T-t)} \frac{1}{T}=\frac{T-s}{T^{2}}, \\
& \lim _{t \rightarrow T-} \Phi(t, s)=\lim _{t \rightarrow T-} \frac{s(T-t)}{t(T-t)} \frac{1}{T}=\frac{s}{T^{2}},
\end{aligned}
$$

thus we can extend $\Phi(t, s)$ continuously to $[0, T]$ and for every $s \in(0, T)$ we have $\Phi(t, s)>0$ for $t \in[0, T]$.

We can define

$$
F(t)=\int_{0}^{T} \Phi(t, s) \mathrm{d} s \text { for } t \in[0, T]
$$

For every $t \in[0, T]$ there exists $d_{0}>0$ such that $d_{0} \leq c F(t)$. From the equation $-u^{\prime \prime}=-u^{\prime \prime}$ we have

$$
\begin{aligned}
u(t) & =-\int_{0}^{T} G(t, s) u^{\prime \prime}(s) \mathrm{d} s \geq \int_{0}^{T} G(t, s) c \mathrm{~d} s \\
& =t(T-t) c \int_{0}^{T} \Phi(t, s) \mathrm{d} s=t(T-t) c F(t) \geq t(T-t) d_{0}, \\
\|u\|_{C} & \geq u\left(\frac{T}{2}\right) \geq \frac{T^{2} d_{0}}{4}=\eta .
\end{aligned}
$$

Lemma 3.3. Let $c, \gamma, \delta>0, \alpha, \beta \in[0,1]$, the functions $\omega_{0}, \omega_{1}, h_{0}, h_{1}, h_{2}$ satisfy assumptions (3.2) and (3.3). Then there exists $r>1$ such that for each $u \in A C^{1}[0, T]$ satisfying (1.2), (3.5) and

$$
\begin{aligned}
-u^{\prime \prime}(t) \leq & \left(\omega_{0}(1)+\omega_{0}(u(t))\right) t^{\gamma}(T-t)^{\delta}+\omega_{1}(1)+\omega_{1}\left(\left|u^{\prime}(t)\right|\right)+h_{0}(t) \\
& +h_{1}(t)\left((u(t))^{\alpha}+1\right)+h_{2}(t)\left(\left|u^{\prime}(t)\right|^{\beta}+1\right)
\end{aligned}
$$

the estimate $\|u\|_{C^{1}} \leq r$ is valid.

Proof. Condition (3.5) implies that $u$ is nonnegative, concave and that there exists $t_{0} \in[0, T]$, such that $u^{\prime}\left(t_{0}\right)=0$.

By Lemma 3.2 there exists $\eta>0$ such that

$$
\begin{aligned}
\eta \frac{t}{T} & \leq \eta \frac{t}{t_{0}} \leq u(t) \quad \text { for } \quad t \in\left[0, t_{0}\right], \\
\eta \frac{T-t}{T} & \leq \eta \frac{T-t}{T-t_{0}} \leq u(t) \quad \text { for } \quad t \in\left[t_{0}, T\right] .
\end{aligned}
$$


Since $\omega_{0}$ is a nonincreasing function, we have

$$
\begin{aligned}
\int_{0}^{T} t^{\gamma}(T-t)^{\delta} & \omega_{0}(u(t)) \mathrm{d} t \leq \int_{0}^{t_{0}} t^{\gamma}(T-t)^{\delta} \omega_{0}(u(t)) \mathrm{d} t+\int_{t_{0}}^{T} t^{\gamma}(T-t)^{\delta} \omega_{0}(u(t)) \mathrm{d} t \\
& \leq \int_{0}^{t_{0}} t^{\gamma}(T-t)^{\delta} \omega_{0}\left(\frac{\eta t}{T}\right) \mathrm{d} t+\int_{t_{0}}^{T} t^{\gamma}(T-t)^{\delta} \omega_{0}\left(\frac{\eta(T-t)}{T}\right) \mathrm{d} t \\
& \leq T^{\delta} \int_{0}^{t_{0}} t^{\gamma} \omega_{0}\left(\frac{\eta t}{T}\right) \mathrm{d} t+T^{\gamma} \int_{t_{0}}^{T}(T-t)^{\delta} \omega_{0}\left(\frac{\eta(T-t)}{T}\right) \mathrm{d} t .
\end{aligned}
$$

Without loss of generality we can assume that $\eta<T$ and, by (3.2), using the substitution $z=\frac{\eta t}{T}$ into the first integral and $T-z=\frac{\eta(T-t)}{T}$ into the second integral we get

$$
\begin{gathered}
\int_{0}^{T} t^{\gamma}(T-t)^{\delta} \omega_{0}(u(t)) \mathrm{d} t \\
\leq T^{\delta} \frac{T}{\eta} \int_{0}^{\frac{\eta t_{0}}{T}}\left(\frac{T z}{\eta}\right)^{\gamma} \omega_{0}(z) \mathrm{d} z+T^{\gamma} \frac{T}{\eta} \int_{T-\frac{\eta\left(T-t_{0}\right)}{T}}^{T}\left(\frac{T}{\eta}(T-z)\right)^{\delta} \omega_{0}(T-z) \mathrm{d} z \\
\leq \frac{T^{\delta+1}}{\eta}\left(\frac{T}{\eta}\right)^{\gamma} \int_{0}^{\frac{\eta t_{0}}{T}} z^{\gamma} \omega_{0}(z) \mathrm{d} z+\frac{T^{\gamma+1}}{\eta}\left(\frac{T}{\eta}\right)^{\delta} \int_{T-\frac{\eta\left(T-t_{0}\right)}{T}}^{T}(T-z)^{\delta} \omega_{0}(T-z) \mathrm{d} z \\
\leq \frac{T^{\gamma+\delta+1}}{\eta^{2}} \int_{0}^{T}\left(z^{\gamma}+z^{\delta}\right) \omega_{0}(z) \mathrm{d} z=A<\infty,
\end{gathered}
$$

where the constant $A$ is independent of the function $u$.

Integrating $c \leq-u^{\prime \prime}$ on $\left[t_{0}, t\right]$ we get

$$
\begin{aligned}
& c\left(t_{0}-t\right) \leq u^{\prime}(t)=\left|u^{\prime}(t)\right| \text { for } t \in\left[0, t_{0}\right], \\
& c\left(t-t_{0}\right) \leq-u^{\prime}(t)=\left|u^{\prime}(t)\right| \text { for } t \in\left[t_{0}, T\right] .
\end{aligned}
$$

Since $\omega_{1}$ is nonincreasing, we have

$$
\begin{aligned}
\int_{0}^{T} \omega_{1}\left(\left|u^{\prime}(t)\right|\right) \mathrm{d} t & =\int_{0}^{t_{0}} \omega_{1}\left(\left|u^{\prime}(t)\right|\right) \mathrm{d} t+\int_{t_{0}}^{T} \omega_{1}\left(\left|u^{\prime}(t)\right|\right) \mathrm{d} t \\
& \leq \int_{0}^{t_{0}} \omega_{1}\left(c\left(t_{0}-t\right)\right) \mathrm{d} t+\int_{t_{0}}^{T} \omega_{1}\left(c\left(t-t_{0}\right)\right) \mathrm{d} t .
\end{aligned}
$$


Without loss of generality we can assume $c<1$ and, by (3.2), substituting $z=c\left(t_{0}-t\right)$ into the first integral and $z=c\left(t-t_{0}\right)$ into second the integral, we get

$$
\begin{aligned}
\int_{0}^{T} \omega_{1}\left(\left|u^{\prime}(t)\right|\right) \mathrm{d} t & \leq \int_{0}^{t_{0}} \omega_{1}\left(c\left(t_{0}-t\right)\right) \mathrm{d} t+\int_{t_{0}}^{T} \omega_{1}\left(c\left(t-t_{0}\right)\right) \mathrm{d} t \\
& =-\frac{1}{c} \int_{c t_{0}}^{0} \omega_{1}(z) \mathrm{d} z+\frac{1}{c} \int_{0}^{c\left(T-t_{0}\right)} \omega_{1}(z) \mathrm{d} z=B<\infty
\end{aligned}
$$

where $B$ is independent of $u$, too.

We set $\max \left\{\left|u^{\prime}(t)\right| ; t \in[0, T]\right\}=\max \left\{\left|u^{\prime}(0)\right| ;\left|u^{\prime}(T)\right|\right\}=\left|u^{\prime}\left(\tau_{0}\right)\right|=\rho$. Then

$$
-\rho T \leq u(t) \leq \rho T \text { for } t \in[0, T] .
$$

Let $C=\omega_{0}(1) \int_{0}^{T} t^{\gamma}(T-t)^{\delta} \mathrm{d} t+\omega_{1}(1) T$. Integrating (3.6) from $\tau_{0}$ to $t_{0}$ we get

$$
\begin{aligned}
\left|u^{\prime}\left(\tau_{0}\right)\right|= & \rho \leq C+\left|\int_{\tau_{0}}^{t_{0}} t^{\gamma}(T-t)^{\delta} \omega_{0}(u(s)) \mathrm{d} s\right|+\left|\int_{\tau_{0}}^{t_{0}} \omega_{1}\left(\left|u^{\prime}(s)\right|\right) \mathrm{d} s\right| \\
& +\left|\int_{\tau_{0}}^{t_{0}} h_{0}(s)+h_{1}(s)\left(|u(s)|^{\alpha}+1\right)+h_{2}(s)\left(\left|u^{\prime}(s)\right|^{\beta}+1\right) \mathrm{d} s\right|
\end{aligned}
$$

and

$$
\rho \leq A+B+C+\left|\int_{\tau_{0}}^{t_{0}} h_{0}(s)+h_{1}(s)\left(|u(s)|^{\alpha}+1\right)+h_{2}(s)\left(\left|u^{\prime}(s)\right|^{\beta}+1\right) \mathrm{d} s\right| .
$$

Hence, since $|u(s)|^{\alpha} \leq|\rho T|^{\alpha},\left|u^{\prime}(s)\right|^{\beta} \leq \rho^{\beta}$ we have

$$
\rho \leq A+B+C+\left|\int_{\tau_{0}}^{t_{0}} h_{0}(s)+h_{1}(s)\left((\rho T)^{\alpha}+1\right)+h_{2}(s)\left(\rho^{\beta}+1\right) \mathrm{d} s\right|
$$

and, consequently,

$$
\rho \leq A+B+C+\left\|h_{0}\right\|_{L}+\left((\rho T)^{\alpha}+1\right)\left\|h_{1}\right\|_{L}+\left(\rho^{\beta}+1\right)\left\|h_{2}\right\|_{L} .
$$

By contradiction we show, that there exists a constant $r^{*}>0$ (independent of $u$ ) such that $\rho<r^{*}$ for every $u$. Assume that there is a sequence $\left\{u_{n}\right\}$ satisfying (1.2), (3.5) and (3.6) and the corresponding sequence $\left\{\rho_{n}\right\}$ is not bounded.

- Let $\alpha, \beta<1$, then from (3.11) we get

$$
1 \leq \frac{A+B+C+\left\|h_{0}\right\|_{L}+\left\|h_{1}\right\|_{L}+\left\|h_{2}\right\|_{L}}{\rho_{n}}+\frac{T^{\alpha}\left\|h_{1}\right\|_{L}}{\rho_{n}^{(1-\alpha)}}+\frac{\left\|h_{2}\right\|_{L}}{\rho_{n}^{(1-\beta)}}
$$

and for $n \rightarrow \infty$ we get

$$
1 \leq 0
$$

which is a contradiction. 
- Let $\alpha=1, \beta<1$, then for $n \rightarrow \infty$ from (3.11) we get

$$
\begin{gathered}
1 \leq \frac{A+B+C+\left\|h_{0}\right\|_{L}+\left\|h_{1}\right\|_{L}+\left\|h_{2}\right\|_{L}}{\rho_{n}}+T\left\|h_{1}\right\|_{L}+\frac{\left\|h_{2}\right\|_{L}}{\rho_{n}^{(1-\beta)}}, \\
1 \leq T\left\|h_{1}\right\|_{L} .
\end{gathered}
$$

By (3.3) we have $T\left\|h_{1}\right\|_{L}<1$ and we have a contradiction.

- Let $\alpha<1, \beta=1$, then for $n \rightarrow \infty$ from (3.11) we get

$$
\begin{gathered}
1 \leq \frac{A+B+C+\left\|h_{0}\right\|_{L}+\left\|h_{1}\right\|_{L}+\left\|h_{2}\right\|_{L}}{\rho_{n}}+\frac{T^{\alpha}\left\|h_{1}\right\|_{L}}{\rho_{n}^{(1-\alpha)}}+\left\|h_{2}\right\|_{L}, \\
1 \leq\left\|h_{2}\right\|_{L} .
\end{gathered}
$$

By (3.3) we have $\left\|h_{2}\right\|_{L}<1$ and we have a contradiction too.

- Let $\alpha=\beta=1$, then

$$
\begin{gathered}
\rho_{n} \leq A+B+C+\left\|h_{0}\right\|_{L}+\left\|h_{1}\right\|_{L}+\left\|h_{2}\right\|_{L}+\rho_{n} T\left\|h_{1}\right\|_{L}+\rho_{n}\left\|h_{2}\right\|_{L}, \\
1 \leq \frac{A+B+C+\left\|h_{0}\right\|_{L}+\left\|h_{1}\right\|_{L}+\left\|h_{2}\right\|_{L}}{\rho_{n}}+T\left\|h_{1}\right\|_{L}+\left\|h_{2}\right\|_{L} .
\end{gathered}
$$

By (3.3) we have $T\left\|h_{1}\right\|_{L}+\left\|h_{2}\right\|_{L}<1$ and thus

$$
1 \leq T\left\|h_{1}\right\|_{L}+\left\|h_{2}\right\|_{L}<1
$$

we again have a contradiction.

Hence there exists $r^{*}>0$ such that $\rho<r^{*}$ for each $u$ satisfying (1.2), (3.5) and (3.6). Since $\|u\|_{C^{1}} \leq \rho T+\rho$, we set $r=r^{*} T+r^{*}+1$.

Proof of Theorem 3.1.

Step 1. Construction of an auxiliary singular problem.

Let $r \in(1, \infty)$ be given by Lemma 3.3. For a.e. $t \in[0, T]$ and for all $x, y, z \in \mathbb{R}$ define the auxiliary functions

$$
\sigma(z)= \begin{cases}z & \text { for }|z| \leq r \\ r \operatorname{sign} z & \text { for }|z|>r\end{cases}
$$

and

$$
g(t, x, y)=f(t,|\sigma(x)|, \sigma(y)) .
$$

We apply Theorems 2.1 and 2.2 to the auxiliary singular problem

$$
-u^{\prime \prime}=g\left(t, u, u^{\prime}\right), \quad u(0)=0, \quad u(T)=0
$$

and we prove that problem (3.12) has a solution $u$ such that

$$
0<u(t) \leq r \text { for } t \in(0, T) \text { and }\left\|u^{\prime}\right\|_{C} \leq r .
$$

Then $u$ is also a solution of problem (1.1), (1.2). 
Step 2. Construction of approximate regular problems.

Since $f$ has no time singularities at $t=0$ and $t=T$, we can put $\Delta_{n}=[0, T]$ for $n \in \mathbb{N}$. Now, choosing an arbitrary $n \in \mathbb{N}$, for a.e. $t \in[0, T]$ and all $x, y \in \mathbb{R}$ we obtain

$$
g_{n}(t, x, y)= \begin{cases}g(t,|x|, y) & \text { if }|x| \geq \frac{1}{n} \\ g\left(t, \frac{1}{n}, y\right) & \text { if }|x|<\frac{1}{n}\end{cases}
$$

and

$$
f_{n}(t, x, y)= \begin{cases}g_{n}(t, x, y) & \text { if }|y| \geq \frac{1}{n} \\ \frac{n}{2}\left[g_{n}\left(t, x, \frac{1}{n}\right)\left(y+\frac{1}{n}\right)-g_{n}\left(t, x,-\frac{1}{n}\right)\left(y-\frac{1}{n}\right)\right] & \text { if }|y|<\frac{1}{n}\end{cases}
$$

We see that $f_{n} \in \operatorname{Car}\left([0, T] \times \mathbb{R}^{2}\right.$ satisfies

$$
\begin{aligned}
& f_{n}(t, x, y)=g(t, x, y) \text { for a.e. } t \in[0, T] \\
& \text { and all } x \in\left[\frac{1}{n}, \infty\right), \quad|y| \in\left[\frac{1}{n}, \infty\right) .
\end{aligned}
$$

Further we have

$c \leq f_{n}(t, x, y) \leq t^{\gamma}(T-t)^{\delta} \omega_{0}\left(\frac{1}{n}\right)+\omega_{1}\left(\frac{1}{n}\right)+h_{0}(t)+h_{1}(t) r^{\alpha}+h_{2}(t) r^{\beta}=m_{n}(t)$

for a.e. $t \in[0, T]$.

Since $m_{n} \in L[0, T]$, Theorem 1.5 yields a solution $u_{n}$ of the problem

$$
-u^{\prime \prime}=f_{n}\left(t, u, u^{\prime}\right), \quad u(0)=0, \quad u(T)=0
$$

for each $n \in \mathbb{N}$.

Step 3. Convergence of a sequence $\left\{u_{n}\right\}$ of approximate solutions.

By (3.4) and (3.14) we get

$$
\begin{gathered}
c \leq-u_{n}^{\prime \prime}(t) \leq t^{\gamma}(T-t)^{\delta}\left(\omega_{0}\left(u_{n}(t)\right)+\omega_{0}(1)\right)+\omega_{1}(1)+\omega_{1}\left(\left|u_{n}^{\prime}(t)\right|\right) \\
+h_{0}(t)+h_{1}(t)\left(u_{n}(t)^{\alpha}+1\right)+h_{2}(t)\left(\left|u_{n}^{\prime}(t)\right|^{\beta}+1\right)
\end{gathered}
$$

for a.e. $t \in[0, T]$ and all $n \in \mathbb{N}$.

Therefore, due to Lemma 3.3,

$$
\left\|u_{n}\right\|_{C^{1}} \leq r \text { for each } n \in \mathbb{N} .
$$

Define the set

$$
\Omega=\left\{x \in C^{1}[0, T]:\|x\|_{C^{1}} \leq r\right\} .
$$

By Theorem 2.1 there exist $u \in C[0, T]$ and a subsequence $\left\{u_{k}\right\} \subset\left\{u_{n}\right\}$ such that (2.3) holds.

Further we have

$$
u_{n}(0)=0, \quad u_{n}(T)=0 \text { and } u_{n}^{\prime \prime}(t)<0 \text { for a.e. } t \in[0, T] .
$$


Therefore $u_{n}>0$ on $(0, T)$ and $u_{n}$ has a unique maximum point $t_{n} \in(0, T)$. By Lemma 3.2, there is $\eta \in\left(0, \frac{r T}{2}\right)$ such that

$$
\begin{gathered}
u_{n}\left(t_{n}\right)>\eta, \quad u_{n}(t) \geq \begin{cases}\frac{\eta t}{T} & \text { for } t \in\left[0, t_{n}\right], \\
\frac{\eta(T-t)}{T} & \text { for } t \in\left[t_{n}, T\right],\end{cases} \\
u_{n}^{\prime}\left(t_{n}\right)=0, \quad c\left(t_{n}-t\right) \leq u_{n}^{\prime}(t) \quad \text { for } t \in\left[0, t_{n}\right],
\end{gathered}
$$

By (3.16) and the Mean Value Theorem for $u_{n}$ on the intervals $\left[0, t_{n}\right]$ and $\left[t_{n}, T\right]$ we get

$$
0<\frac{\eta}{r} \leq t_{n} \leq T-\frac{\eta}{r}<T, \quad n \in \mathbb{N},
$$

and we can choose a subsequence $\left\{u_{k}\right\}$ in such a way that it satisfies (2.3) and $\lim _{k \rightarrow \infty} t_{k}=t_{0} \in(0, T)$. Then

$$
u(t) \geq \begin{cases}\frac{\eta t}{T} & \text { for } t \in\left[0, t_{0}\right] \\ \frac{\eta(T-t)}{T} & \text { for } t \in\left[t_{0}, T\right] .\end{cases}
$$

Put $\Sigma=\left\{t_{0}\right\}$ and choose an arbitrary interval $[a, b] \subset(0, T) \backslash \Sigma$. For example, let $[a, b] \subset\left(0, t_{0}\right)$. Then there exists $k_{0} \in \mathbb{N}$ such that for $k \geq k_{0}$ we have

$$
\begin{gathered}
\left|t_{k}-t_{0}\right| \leq \frac{1}{2}\left(t_{0}-b\right), \quad[a, b] \subset\left(\frac{1}{k}, t_{k}\right), \\
u_{k}(t) \geq \frac{\eta a}{T}=m_{0}, \quad u_{k}^{\prime}(t) \geq c\left(t_{k}-t\right) \geq c\left(t_{k}-b\right) \geq \frac{1}{2}\left(t_{0}-b\right)=m_{1}
\end{gathered}
$$

for $t \in[a, b]$. If we choose $[a, b] \subset\left(t_{0}, T\right)$, we argue similarly.

Thus, for a.e. $t \in[a, b]$

$$
\left|f_{k}\left(t, u_{k}(t), u_{k}^{\prime}(t)\right)\right| \leq \psi(t) \in L[a, b],
$$

where

$$
\psi(t)=\sup \left\{\left|f(t, x, y): \quad m_{0} \leq x \leq r, \quad m_{1} \leq\right| y \mid \leq r\right\} .
$$

We have proved that on each $[a, b] \subset(0, T) \backslash \Sigma$ there exists $\psi \in L[a, b]$ such that

$$
\left|u_{k}^{\prime \prime}(t)\right| \leq \psi(t) \text { for a.e. } t \in[a, b] \text { and all } k \in \mathbb{N}, \quad k \geq k_{0} .
$$

By virtue of the absolute continuity of the Lebesgue integral we see that the sequence $\left\{u_{k}^{\prime}\right\}$ satisfies $(2.4)$ and, by Theorem $2.1, u \in C^{1}((0, T) \backslash \Sigma)$ and $(2.5)$ is valid.

Step 4. The function $u$ is a solution of problem (1.1), (1.2).

Conditions (2.5) and (3.17) imply

$$
\begin{array}{ll}
c\left(t_{0}-t\right) \leq u^{\prime}(t) & \text { for } t \in\left(0, t_{0}\right), \\
c\left(t-t_{0}\right) \leq-u^{\prime}(t) & \text { for } t \in\left(t_{0}, T\right) .
\end{array}
$$

Since $u_{k}^{\prime}$ is decreasing on $[0, T]$ for each $k \geq k_{0}, u^{\prime}$ is noincreasing on $\left(0, t_{0}\right)$ and on $\left(t_{0}, T\right)$. Therefore there exist the limits

$$
\lim _{t \rightarrow t_{0}-} u^{\prime}(t), \quad \lim _{t \rightarrow t_{0}+} u^{\prime}(t)
$$


and

$$
\lim _{t \rightarrow 0+} u^{\prime}(t) \geq c t_{0}>0, \quad \lim _{t \rightarrow T-} u^{\prime}(t) \leq-c\left(T-t_{0}\right)<0 .
$$

We summarize that $u^{\prime}(t)>0$ on $\left[0, t_{0}\right)$ and $u^{\prime}(t)<0$ on $\left(t_{0}, T\right]$. So, $t_{0}$ is the unique point where $u^{\prime}\left(t_{0}\right)=0$ or $u^{\prime}\left(t_{0}\right)$ does not exist. By (3.18), $u$ is positive on $(0, T)$. Hence $\Sigma=\left\{t_{0}\right\}$ satisfies $(2.8)$ and, by Theorem 2.2, $u \in A C_{l o c}^{1}((0, T) \backslash \Sigma)$ is a $w$-solution of problem (3.12). Finally, by (3.4), we have $f_{k}\left(t, u_{k}(t), u_{k}^{\prime}(t)\right) \geq$ 0 for a.e. $t \in[0, T]$ and all $k \in \mathbb{N}$. Hence (2.9) holds and, by Theorem 2.2, $u$ is a solution of problem (3.12). Having in mind that $u>0$ on $(0, T)$ and $\left\|u_{k}\right\|_{C^{1}} \leq r$ hold, we get by (2.3) and (2.5) that estimate (3.13) is satisfied and thus $u$ is a solution of problem (1.1), (1.2).

Example 3.4. Let $h_{1}, h_{2} \in L[0, T]$ be nonnegative. For a.e. $t \in[0, T]$ and all $x, y \in \mathbb{R}$ define the function

$$
f(t, x, y)=1+\frac{t^{\frac{3}{2}}(T-t)^{\frac{3}{2}}}{x^{2}}+h_{1}(t) \sqrt{x}+\frac{1}{\sqrt{|y|}}\left(1+h_{2}(t)|y|\right) .
$$

The second term of $f$ has a space singularity at $x=0$ and the last one a singularity at $y=0$. We can check that $f$ satisfies the conditions of Theorem 3.1 with $h_{0}(t) \equiv c=1, \alpha=\beta=\frac{1}{2}, \gamma=\delta=\frac{3}{2}, \omega_{0}(x)=\frac{1}{x^{2}}$ and $\omega_{1}(|y|)=\frac{1}{\sqrt{|y|}}$.

Example 3.5. Let $T=1$. For a.e. $t \in[0,1]$ and all $x, y \in \mathbb{R}$ define the function

$$
f(t, x, y)=\sqrt{1-t}\left(1+\frac{t^{2}}{x}\right)+\frac{3}{\sqrt[3]{|y|}}+\frac{1}{6 \sqrt{t}}(x+|y|) .
$$

The first term has space singularity at $x=0$ and the second one at $y=0$. We see that $f$ satisfies the conditions of Theorem 3.1 if we put $\alpha=\beta=1, \gamma=2$, $\delta=\frac{1}{2}, \omega_{0}(x)=\frac{1}{x}, \omega_{1}(|y|)=\frac{3}{\sqrt[3]{|y|}}, h_{0}(t)=\sqrt{1-t}, h_{1}(t)=\frac{1}{6 \sqrt{t}}, h_{2}(t)=\frac{1}{6 \sqrt{t}}$, and choose $c>0$ sufficiently small.

Example 3.6. Let $T=2 \pi$. We define the function $f$ for a.e. $t \in[0,2 \pi]$ and all $x, y \in \mathbb{R}$ by

$$
f(t, x, y)=t \sqrt[5]{t^{3}}\left(e+10 \frac{\sqrt[3]{(2 \pi-t)^{4}}}{x^{2}}\right)+\frac{e}{\sqrt[5]{|y|}}+t^{3} \sqrt[6]{x}+\frac{5 t^{4}+2 t}{10000}|y|
$$

The function $f$ has space singularities at $x=0$ and $y=0$. We can check that $f$ satisfies the assumptions of Theorem 3.1 for $\alpha=\frac{1}{6}, \beta=1, \gamma=\frac{8}{5}, \delta=\frac{4}{3}$, $\omega_{0}(x)=\frac{10}{x^{2}}, \omega_{1}(|y|)=\frac{e}{\sqrt[5]{|y|}}, h_{0}(t)=e t \sqrt[5]{t^{3}}, h_{1}(t)=t^{3}, h_{2}(t)=\frac{5 t^{4}+2 t}{10000}$ and sufficiently small $c$.

\section{ACKNOWLEDGEMENTS}

This research was supported by the Council of Czech Government MSM6198959214 and by the grant No. A100190703 of the Grant Agency of the Academy of Sciences of the Czech Republic. 


\section{REFERENCES}

1. R. P. Agarwal, H. Lü, and D. O'Regan, An upper and lower solution method for the one-dimensional singular p-Laplacian. Mem. Differential Equations Math. Phys. 28(2003), $13-31$.

2. R. P. Agarwal and D. O'Regan, Singular boundary value problems for superlinear second order ordinary and delay differential equations. J. Differential Equations 130(1996), No. 2, 333-355.

3. R. P. Agarwal and D. O'Regan, Nonlinear superlinear singular and nonsingular second order boundary value problems. J. Differential Equations 143(1998), No. 1, 60-95.

4. R. P. Agarwal and D. O'Regan, A survey of recent results for initial and boundary value problems singular in the dependent variable. Handbook of differential equations, 1-68, Elsevier/North-Holland, Amsterdam, 2004.

5. R. P. Agarwal, D. O'Regan, V. Lakshmikantham, and S. Leela, Existence of positive solutions for singular initial and boundary value problems via the classical upper and lower solution approach. Nonlinear Anal., Ser. A: Theory Methods 50(2002), No. 2, $215-222$.

6. R. G. Bartle, A modern theory of integration. Graduate Studies in Mathematics, 32. American Mathematical Society, Providence, RI, 2001.

7. J. V. BaXley, Some singular nonlinear boundary value problems. SIAM J. Math. Anal. 22(1991), No. 2, 463-479.

8. P. HABETS and F. ZANolin, Upper and lower solutions for a generalized Emden-Fowler equation. J. Math. Anal. Appl. 181(1994), No. 3, 684-700.

9. J. K. Hunter and B. Nachtergaele, Applied analysis. World Scientific Publishing Co., Inc., River Edge, NJ, 2001.

10. D. Q. Jiang, Upper and lower solutions method and a superlinear singular boundary value problem. Comput. Math. Appl. 44(2002), No. 3-4, 323-337.

11. I. T. Kiguradze, Some singular boundary value problems for ordinary differential equations. (Russian) Izdat. Tbilis. Univ., Tbilisi, 1975.

12. I. T. Kiguradze and B. L. SheKhter, Singular boundary value problems for secondorder ordinary differential equations. (Russian) Itogi Nauki i Tekhniki, Current problems in mathematics. Newest results, Vol. 30 (Russian), 105-201, 204, Akad. Nauk SSSR, Vsesoyuz. Inst. Nauchn. i Tekhn. Inform., Moscow, 1987; English transl.: J. Soviet Math. 43(1988), No. 2, 2340-2417.

13. A. Lomtatidze, Positive solutions of boundary value problems for second-order ordinary differential equations with singularities. (Russian) Differentsial'nye Uravneniya 23(1987), No. 10, 1685-1692, 1834-1835; English transl.: Differential Equations 23(1987), 11461152.

14. A. Lomtatidze and P. Torres, On a two-point boundary value problem for second order singular equations. Czechoslovak Math. J. 53(128)(2003), No. 1, 19-43.

15. D. O'Regan, Theory of singular boundary value problems. World Scientific Publishing Co., Inc., River Edge, NJ, 1994.

16. I. RACHŮnKovÁ and S. StANĚK, Sign-changing solutions of singular Dirichlet boundary value problems. Arch. Inequal. Appl. 1(2003), No. 1, 11-29.

17. I. RAChŮnková and S. StaněK, Connections between types of singularities in differential equations and smoothness of solutions for Dirichlet BVPs. Second International Conference on Dynamics of Continuous, Discrete and Impulsive Systems (London, ON, 2001). Dyn. Contin. Discrete Impuls. Syst. Ser. A Math. Anal. 10(2003), No. 1-3, 209222. 
18. I. RachŮnková and S. StanĚK, General existence principle for singular BVPs and its application. Georgian Math. J. 11(2004), No. 3, 549-565.

19. G. E. Shilov and B. L. Gurevich, Integral, measure and derivative: a unified approach. (Translated from the Russian) Revised English edition. Dover Books on Advanced Mathematics. Dover Publications, Inc., New York, 1977.

20. S. StanĚK, Positive solutions of singular positone Dirichlet boundary value problems. Math. Comput. Modelling 33(2001), No. 4-5, 341-351.

21. S. D. TAliaferro, A nonlinear singular boundary value problem. Nonlinear Anal. 3(1979), No. 6, 897-904.

22. A. Tineo, Existence theorems for a singular two-point Dirichlet problem. Nonlinear Anal. 19(1992), No. 4, 323-333.

23. N. I. VASIL'Ev and Ju. A. Klokov, Foundations of the theory of boundary value problems in ordinary differential equations. (Russian) Zinatne, Riga, 1978.

(Received 9.02.2007)

Authors' addresses:

I. Rachůnková

Department of Mathematics

Palacký University

Tomkova 40, 77146 Olomouc

Czech Republic

E-mail: rachunko@inf.upol.cz

J. Stryja

Department of Mathematics and Descriptive Geometry

VŠB - Technical University of Ostrava

17. listopadu 15, 70833 Ostrava-Poruba

Czech Republic

E-mail: jakub.stryja@vsb.cz 